\title{
ATTÉNUATION ET VITESSE ULTRASONORES CRITIQUES AU-DESSOUS D'UNE TRANSITION SMECTIQUE A-CHOLESTÉRIQUE FAIBLEMENT DU PREMIER ORDRE
}

\author{
J. C. BACRI \\ Laboratoire d'Ultrasons $(*)$, Université Paris VI, \\ Tour 13, 4, place Jussieu, 75230 Paris Cedex 05, France
}

(Reçu le 26 mai 1975, accepté le 20 juin 1975)

\begin{abstract}
Résumé. - Nous avons mesuré l'atténuation et la vitesse ultrasonores au voisinage d'une transition smectique A-cholestérique faiblement du premier ordre à $560 \mathrm{MHz}$ et $1000 \mathrm{MHz}$. Nous avons interprété les résultats pour $T<T_{\mathrm{c}}$ en utilisant l'analogie avec la transition $\lambda$ de l'hélium.

Abstract. - Ultrasonic attenuation and velocity were measured at $560 \mathrm{MHz}$ and $1000 \mathrm{MHz}$ near a weakly first order smectic A-cholesteric phase transition. The results are explained for $T<T_{\mathrm{c}}$ by analogy to the $\lambda$ transition.
\end{abstract}

1. Introduction. - De nombreuses expériences [1-9] ont mis en évidence les phénomènes critiques au voisinage d'une transition faiblement du premier ordre smectique A-nématique d'un cristal liquide. La plupart des mesures concernent les phénomènes prétransitionnels de certaines constantes de Frank et de quelques coefficients de viscosité dans la phase haute température.

De Gennes [10a] a montré que dans la phase cholestérique au voisinage d'une transition smectique A-cholestérique, le pas avait un comportement critique, analogue aux constantes de Frank $K_{2}$ et $K_{3}$ dans une transition $S_{A} \leftrightarrow N$.

Un effet prétransitionnel sur le pas a été observé [7]; l'exposant critique de la divergence du pas en fonction de la température est $v=0,675 \pm 0,025$ : cet exposant est en accord avec la théorie de De Gennes [11] qui admet l'analogie entre la transition $\lambda$ de l'hélium et la transition smectique A-nématique.

Lubensky $[10 b]$ a démontré que la transition smectique A-cholestérique est similaire à la transition supraconductrice sous champ magnétique et qu'elle est, par conséquent, toujours du premier ordre, si on la compare à un supraconducteur de type I

$$
\left(K=\lambda / \xi<2^{-1 / 2}\right) \text {. }
$$

$\mathrm{Si}$ on appelle $\Delta T_{1}$ la variation de la température de transition entre une transition smectique $\mathrm{A}$-nématique (du second ordre) et une transition smectique A-cholestérique, Lubensky a trouvé que $\Delta T_{1} \sim 0,1^{\circ} \mathrm{C}$.

(*) Equipe de Recherche Associée au C.N.R.S.
Donc on peut avoir, dans certains cas, des transitions smectiques A-cholestériques faiblement du premier ordre. Si on admet l'analogie entre une transition $\mathrm{S}_{\mathrm{A}} \leftrightarrow$ Ch et une transition supraconductrice de type II $\left(K>2^{-1 / 2}\right)$ sous champ magnétique, on aura une transition du second ordre.

Dans cette lettre, nous décrivons les effets critiques de l'atténuation et de la vitesse ultrasonores au voisinage d'une transition smectique A-cholestérique faiblement du premier ordre; ils sont observés dans la phase smectique $\mathrm{A}$.

2. Méthode expérimentale. - La méthode a déjà été présentée [12]. L'appareillage est un système classique d'impulsions utilisé en transmission. Les transducteurs ultrasonores employés sont des barreaux de quartz en coupe $X$ excités par des cavités. Le cristal liquide est déposé entre les deux quartz préalablement traités pour obtenir le directeur parallèle au vecteur d'onde ultrasonore. La température de l'ensemble est régulée à $0,01{ }^{\circ} \mathrm{C}$. La mesure de l'intensité du faisceau ultrasonore transmis donne l'atténuation ultrasonore, la variation de la phase du signal reçu permet d'accéder à la variation de vitesse dans le cristal liquide en fonction d'un paramètre extérieur, ici la température.

Le cristal liquide utilisé est le cholestéryl nonanoate qui a une transition smectique A-nématique faiblement du premier ordre avec une entropie de transition de $0,05 R_{0}$ [13] et une température de transition de $74^{\circ} \mathrm{C}$. Nous avons choisi ce corps parce que d'une part la divergence du pas du cholestérique a été 
observée [7] et d'autre part les effets critiques sur l'atténuation sont très importants; l'erreur commise sur l'exposant critique dû à l'incertitude du fond continu sera donc faible.

3. Résultats expérimentaux. - Nous avons mesuré simultanément les variations de l'atténuation et de la vitesse ultrasonores en fonction de la température dans la phase basse température à $560 \mathrm{MHz}$ et $1000 \mathrm{MHz}$, avec des épaisseurs respectives d'échantillons de $20 \mu$ et $2,5 \mu$. Nous avons porté sur les figures 1 et 2 l'atténuation et la variation de vitesse en fonction de $\Delta T=T-T_{\mathrm{c}}$ aux deux fréquences étudiées.

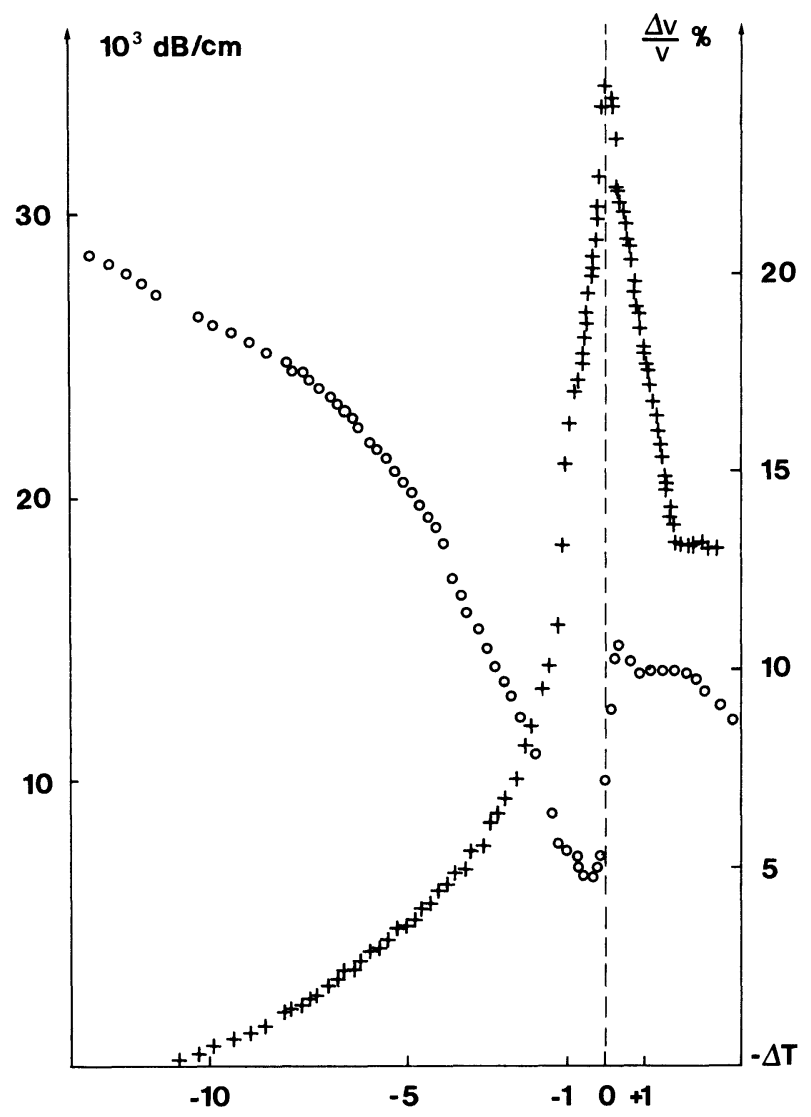

FIG. 1. - Atténuation (croix) et vitesse (cercles) ultrasonores au voisinage de $T_{\mathrm{c}}$ à $560 \mathrm{MHz}$.

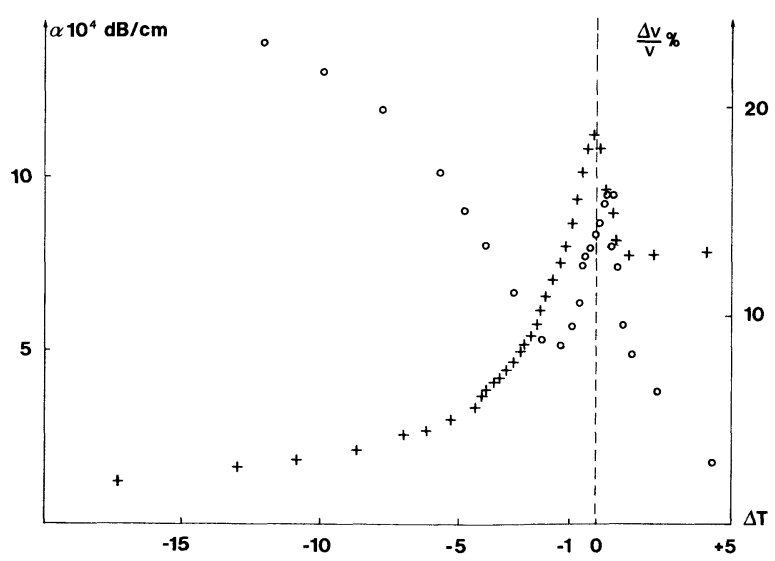

FIG. 2. - Atténuation (croix) et vitesse (cercles) ultrasonores au voisinage de $T_{\mathrm{c}}$ à $1000 \mathrm{MHz}$.
On remarque une forte divergence de l'atténuation à la transition principalement dans la phase basse température. L'atténuation ultrasonore loin de $T_{\mathrm{c}}$ dans la phase cholestérique nous montre que le fond continu ne varie presque pas avec la température; par contre, il n'en est pas de même avec la variation de vitesse. Il est bien connu que la recherche d'un exposant critique est fortement dépendant du fond continu.

On observe une anomalie dans la variation de vitesse. Un minimum pour $\Delta v / v$ apparaît dans la phase smectique $\mathrm{A}$ un peu en-dessous de $T_{\mathrm{c}}$; la position de ce minimum est repérée par $\Delta T_{\mathrm{m}}$

$$
\begin{aligned}
& \Delta T_{\mathrm{m}}=-(0,6 \pm 0,1){ }^{\circ} \mathrm{C} \text { à } \quad 560 \mathrm{MHz} \\
& \Delta T_{\mathrm{m}}=-(1,2 \pm 0,2){ }^{\circ} \mathrm{C} \text { à } 1000 \mathrm{MHz} .
\end{aligned}
$$

4. Interprétation des résultats. - 4.1 ATTÉNUATION ULTRASONORE. - F. Brochard et F. Jähnig [14], en utilisant l'analogie avec la transition $\lambda$ de l'hélium, ont montré que certains coefficients de viscosité au-dessus d'une transition du second ordre smectique A-nématique comportaient une partie critique due aux fluctuations du paramètre d'ordre

$$
\tilde{\eta}(\omega)=\frac{\tilde{\eta}(0)}{1+\omega^{2} \tau^{2}} \quad \text { avec } \quad \tilde{\eta}(0) \sim \frac{\tau(T)}{\xi(T)}
$$

et $\tau(T) \sim \xi^{3 / 2}(T) \quad \tau$ temps de relaxation du para-

$$
\xi(T) \sim(\Delta T / T)^{-2 / 3} \quad \xi \text { longueur de cohérence. }
$$

Il a été montré que la partie critique de l'atténuation se met sous la forme :

$$
\tilde{\alpha}_{\|} \sim \tilde{\eta}_{1}^{\mathrm{MPP}} \frac{\tau}{\xi} \frac{\omega^{2}}{1+\omega^{2} \tau^{2}} .
$$

L'expression ci-dessus a été vérifiée expérimentalement dans deux cristaux liquides $[15,16]$.

Pour $T<T_{\mathrm{NA}}$, Jähnig [17] a montré que les viscosités critiques ont la même dépendance en température que pour $T>T_{\mathrm{NA}}$. Donc l'atténuation critique $\delta \alpha_{\|}$s'écrira :

$$
\delta \alpha_{\|}(\omega) \sim \frac{\tau}{\xi} \frac{\omega^{2}}{1+\omega^{2} \tau^{2}} \sim \frac{\omega^{2}}{1+\omega^{2} \tau_{0}^{2} \varepsilon^{2}} \varepsilon^{-1 / 3}
$$

avec $\varepsilon=\Delta T / T$.

A $560 \mathrm{MHz}$, nous avons $q \xi=1$ pour $\Delta T \simeq 0,1{ }^{\circ} \mathrm{C}$. Dans l'interprétation des résultats expérimentaux, il faut donc tenir compte de la dépendance de l'atténuation en fonction du vecteur d'onde.

En utilisant

$$
\begin{aligned}
\xi(q)=\frac{\xi(0)}{Q^{1 / 2}} \text { avec } \quad \xi(0) & =\xi_{0} \varepsilon^{-2 / 3} \\
Q & =1+q^{2} \xi_{0}^{2} \varepsilon^{-4 / 3}
\end{aligned}
$$


on trouve que

$$
\frac{\delta \alpha_{\|}(q, \omega)}{\omega^{2}} \sim \frac{\varepsilon^{-1 / 3}}{Q^{1 / 4}} \times\left|1+\frac{\omega^{2} \tau_{0}^{2} \varepsilon^{-2}}{Q^{3 / 2}}\right|^{-1} .
$$

Si on considère qu'il y a analogie entre la transition smectique A-nématique et la transition smectique A-cholestérique, dans le cas de transition faiblement du premier ordre, l'atténuation critique sera donnée par l'expression (1).

(Dans $\delta \alpha_{\|}$intervient aussi la perméation, mais à un ordre supérieur $\left(^{1}\right)$.)

Sur les figures 3 et 4 nous avons porté l'atténuation critique (courbes expérimentales et théoriques d'après l'expression (1)) en fonction de $\Delta T$ en échelle logarithmique à $560 \mathrm{MHz}$ et $1000 \mathrm{MHz}$.

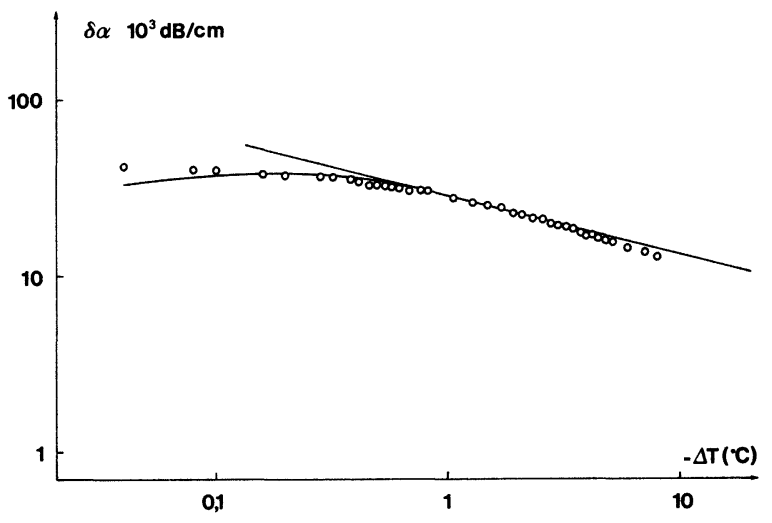

Fig. 3. - Partie critique de l'atténuation en fonction de $\Delta T$ et courbe théorique d'après l'expression (1), à $560 \mathrm{MHz}$.

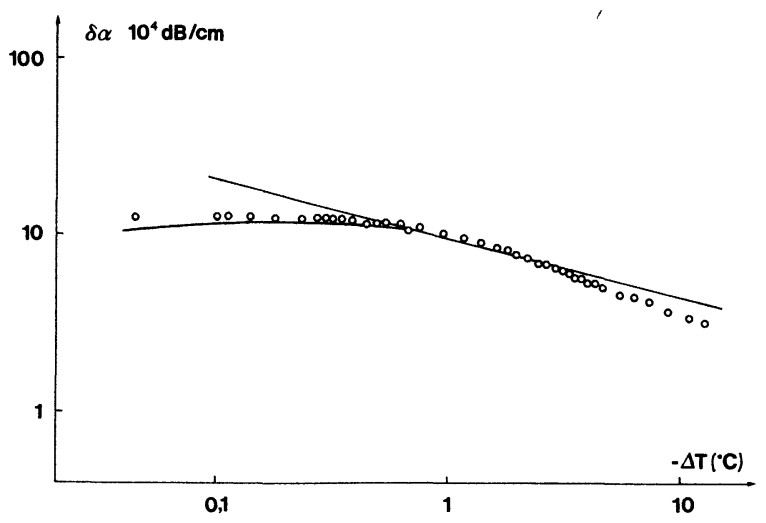

Fig. 4. - Partie critique de l'atténuation en fonction de $\Delta T$ et courbe théorique d'après l'expression (1), à $1000 \mathrm{MHz}$.

Le meilleur accord pour ces deux courbes a été obtenu en prenant comme seul paramètre ajustable $\tau_{0}=10^{-13} \mathrm{~s}$. La dépendance en fréquence des courbes 3 et 4 est bien celle attendue théoriquement.

4.2 VitesSe UltrasonORE. - Pour calculer la dépendance de la vitesse en fonction de la température

(1) Jähnig, F., communication privée. on peut partir de l'expression de l'énergie dans le smectique A [18] :

$$
E=\frac{1}{2} A_{0} \theta^{2}+\frac{1}{2} B_{0} \psi_{0}^{2} q_{\mathrm{s}}^{2}\left(\frac{\partial u}{\partial z}\right)^{2}+C_{0} \psi_{0}^{2} q_{\mathrm{s}} \theta \frac{\partial u}{\partial z}
$$

$A_{0}, B_{0}, C_{0}$ sont les coefficients de rigidité adiabatique, $\theta$ la dilatation, $u$ la position de la couche.

En négligeant l'amortissement et la perméation et dans le cas où le vecteur d'onde est perpendiculaire aux couches, on trouve pour la vitesse du premier son :

$$
\rho v_{\|}^{2}=A_{0}^{2}+B_{0} \psi_{0}^{2} q_{\mathrm{s}}^{2}+C_{0} \psi_{0}^{2} q_{\mathrm{s}} .
$$

Comme dans la plupart des cas on a $B_{0} \gg C_{0}$ $[19,20]$, on gardera ici

$$
\rho v_{\|}^{2}=A_{0}+B_{0} \psi_{0}^{2} q_{\mathrm{s}}^{2}
$$

D'autre part, on sait que dans le cas d'un phénomène à un seul temps de relaxation, la vitesse et l'atténuation des ondes ultrasonores sont données par les relations suivantes :

et

$$
\delta \alpha_{\|}(\omega)=\frac{\Delta v_{\|}}{v_{\|}^{2}(0)} \frac{\omega^{2} \tau}{1+\omega^{2} \tau^{2}}
$$

$$
v_{\|}(\omega)=v_{\|}(0)+\Delta v_{\|} \frac{\omega^{2} \tau^{2}}{1+\omega^{2} \tau^{2}}
$$

avec

$$
\Delta v_{\|}=v_{\|}(\infty)-v_{\|}(0)
$$

$v_{\|}(\omega)$ est la vitesse du premier son à la fréquence $\omega$ : et $v_{\|}(0)$ est donnée par l'expression (2).

D'après (1) la partie critique de l'atténuation peut s'écrire

$$
\delta \alpha_{\|}(\omega)=\frac{\alpha_{0} \omega^{2} \varepsilon^{-1 / 3}}{1+\omega^{2} \tau^{2}}
$$

En comparant (3) et (5) on obtient la valeur de $\Delta v_{\|}$et en remplaçant dans (4) :

$$
\begin{aligned}
v_{\|}(\omega)=\left(A+B \varepsilon^{2 / 3}\right)^{1 / 2}+ & \\
& +\frac{\omega^{2} \alpha_{0} \tau_{0} \varepsilon^{-4 / 3}}{\omega^{2} \tau_{0}^{2} \varepsilon^{-2}}\left(A+B \varepsilon^{2 / 3}\right)
\end{aligned}
$$

et

$$
\begin{aligned}
v_{\|}(\omega, q) & =\left(A+B Q^{1 / 2} \varepsilon^{2 / 3}\right)^{1 / 2}+ \\
& +\frac{\omega^{2} \alpha_{0} \tau_{0} \varepsilon^{-4 / 3} Q^{-1}\left(A+B Q^{1 / 2} \varepsilon^{2 / 3}\right)}{1+\omega^{2} \tau_{0}^{2} \varepsilon^{-2} Q^{-3 / 2}}
\end{aligned}
$$

avec $A=A_{0} / \rho$

$$
B \dot{\varepsilon}^{2 / 3}=B_{0} \psi_{0}^{2} q_{\mathrm{s}}^{2} / \rho .
$$

La vitesse du son définie par l'expression (7) décroît quand on s'approche de la transition, passe par un minimum et commence à croître. Expérimentalement, 
on trouve une dépendance en température analogue. Les minima coïncident en prenant comme valeur numérique :

$$
\begin{aligned}
& A / \rho=2 \times 10^{6} \mathrm{~m}^{2} / \mathrm{s}^{2} . \\
& B / \rho=0,3 \times 10^{6} \mathrm{~m}^{2} / \mathrm{s}^{2} .
\end{aligned}
$$

Les autres valeurs numériques étant identiques à celles utilisées dans l'expression (1). La valeur prise pour $\alpha_{0}$ est cinq fois plus petite que celle donnée par l'atténuation en utilisant l'expression (5).

Loin de la transition, dans l'expression (6), le second terme est faible par rapport au premier :

$$
v_{\|}(\omega)=\left(A+B \varepsilon^{2 / 3}\right)^{1 / 2}
$$

et comme $A \gg B$

$$
\frac{\delta v_{\|}(\omega)}{v_{\|}(\omega)} \sim \varepsilon^{2 / 3}=\left(\frac{\Delta T}{T}\right)^{0,66}
$$

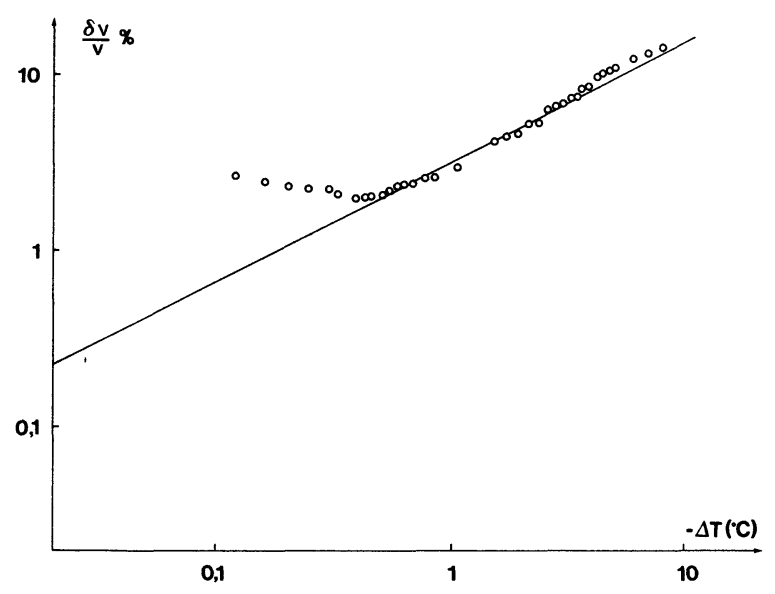

Fig. 5. - Partie critique de la vitesse en fonction de $\Delta T$. La pente de la droite est 0,66 .
Nous avons tracé sur la figure 5 en échelle logarithmique la variation de vitesse $\delta v_{\|} / v_{\|}$en fonction de la température à $560 \mathrm{MHz}$. On remarque que pour $|\Delta T|>0,6^{\circ} \mathrm{C}$ les points expérimentaux sont assez proches d'une droite ayant une pente $\frac{2}{3}$. Le fond continu utilisé est la tangente à la courbe $\Delta v / v$ loin de la transition.

5. Conclusion. - Nous avons observé une importante divergence de l'atténuation et de la vitesse des ondes ultrasonores dans la phase smectique $\mathrm{A}$ du cholestéryl nonanoate au voisinage de la transition $\mathrm{S}_{\mathrm{A}} \leftrightarrow \mathrm{Ch}$. Le meilleur accord entre les courbes théoriques et expérimentales a été obtenu en prenant les mêmes exposants que ceux du point $\lambda$ de l'hélium (et non pas ceux de la théorie du champ moyen). Cette interprétation concorde avec les résultats de Pindak et al. [7] sur la divergence du pas dans la phase cholestérique du même composé en admettant la symétrie des effets de fluctuation du paramètre d'ordre.

Les exposants utilisés seraient compatibles avec une transition du second ordre (en faisant l'analogie avec une transition supraconductrice de type II sous champ magnétique). Dans le cas de l'analogie avec un supradonducteur de type II, la largeur de la phase intermédiaire peut être faible $\Delta T \sim 10^{-2}{ }^{\circ} \mathrm{C}$ pour $K \sim 1$ (dans le CBOOA, $\lambda \simeq 20 \AA$ [22], $\xi_{0} \simeq 20 \AA$ ). Mais la valeur de l'entropie de transition, faible mais non nulle, indique une transition faiblement du premier ordre (analogie avec une transition supraconductrice de type I sous champ magnétique). Il faut aussi tenir compte des effets des impuretés dans la recherche des exposants critiques comme l'a montré Cladis [21].

\section{Bibliographie}

[1] Cheung, L., Meyer, R. B. and Gruler, H., Phys. Rev. Lett. 31 (1973) 349.

[2] Delaye, M., Ribotta, R. and Durand, G., Phys. Rev. Lett. 31 (1973) 443.

[3] Leger, L., Phys. Lett. 44A (1973) 535.

[4] Cladis, P. E., Phys. Rev. Lett. 31 (1973) 1200.

[5] Cheung, L., and Meyer, R. B., Phys. Lett. 43A (1973) 261.

[6] Hardouin, F., Achard, M. F., Gasparoux, H., Solid. State Commun. 14 (1974) 453.

[7] Huang, C. C., Pindak, R. S., Flanders, P. J. and Ho, J. T., Phys. Rev. Lett. 33 (1974) 400.

[8] Leger, L. and D'Humières, D., J. Physique Colloq. 36 (1975) C1-113.

[9] Salin, D., Smith, I. W. et Durand, G., J. Physique Lett. 35 (1974) L-165.

[10a] De Gennes, P. G., Mol. Cryst. Liq. Cryst. 21 (1973) 39. [10b] Lubensky, J. C., J. Physique Colloq. 36 (1975) C1-151.

[11] De Gennes, P. G., Solid State Commun. 10 (1972) 753.

[12] BACRI, J. C., J. Physique 35 (1974) 601.

[13] Mc Millan, W. L., Phys. Rev. A 6 (1972) 936.

[14] Brochard, F. et JähnIG, F., J. Physique 35 (1974) 301.

[15] BACRI, J. C., J. Physique Colloq. 36 (1975) C1-123.

[16] BACRI, J. C., J. Physique Lett. 36 (1975) L-177.

[17] JÄHNIG, F., J. Physique 36 (1975) 315.

[18] Brochard, F., J. Physique 34 (1973) 411.

[19] Liao, Y., Clark, N. A., Pershan, P. S., Phys. Rev. Lett. 30 (1973) 639.

[20] Miyano, K. and Ketterson, J. B., Phys. Rev. Lett. 31 (1973) 1047.

[21] Cladis, P. E., Phys. Lett. 48A (1974) 179.

[22] Delaye, M., Ribotta, R. and Durand, G., Phys. Lett. 44A (1973) 139. 